\title{
ADVANCES ON PROGNOSTICS FOR INTELLIGENT MAINTENANCE SYSTEMS
}

\author{
Hai Qiu ${ }^{1}$, Jay Lee ${ }^{1}$, Dragan Djudjanovic ${ }^{2}$, Jun $\mathrm{Ni}^{2}$ \\ NSF Center forIntelligent Maintenance Systems \\ University of Wisconsin-Milwaukee ${ }^{1}$ \\ University of Michigan ${ }^{2}$
}

\begin{abstract}
An increasing number of manufacturers are beginning to realize the importance of adopting new maintenance technologies to enable products and systems to achieve near-zero downtime. Prognostic technology enables this paradigm shift from the traditional "fail and fix" maintenance practices to a "predict and prevent" paradigm. This paper addresses the current issues of prognostic applications in machinery maintenance, and presents an overall prognostic architecture called Intelligent Maintenance Systems (IMS). Discussion is focused on four aspects, including the relationship between diagnostics and prognostics, feature extraction methods, performance assessment techniques, and prediction algorithms. Copyright $@ 2005$ IFAC
\end{abstract}

Keywords: Prediction, Intelligent Manufacturing Systems, Fault Diagnosis, Sensor Fusion

\section{INTRODUCTION}

Both diagnostics and prognostics originally come from the medical field. As the field of maintenance technology emerged, diagnostics and prognostics gradually permeated all areas of mechanical engineering. Diagnostics is only a reactive basis for maintenance decisions. In order to reduce the maintenance cost and maintain the machine uptime at the highest level, maintenance is expected to be carried out in a more proactive way. Therefore, as an emerging technology, prognostics started to attract more and more attention and are gradually implemented in industries. However, there is still no clear and well-recognized definition of machinery prognostics, and a systematic methodology of how to deploy it. The ultimate goal of prognostics should be to detect the early onset of failure conditions, forecast the remaining useful life of machinery or machine components, and assist to make maintenance decision for impending failures. This paper reviews the state of the art of prognostic technology and presents a systematic methodology of how to conduct prognostics in machinery maintenance.

\section{DIAGNOSTICS VERSUS PROGNOSTICS}

Though the knowledge bases used for diagnosis and prognosis may be similar, the way each is implemented is significantly different. Clarifying the relationship between diagnostics and prognostics will greatly help to understand the fundamentals of prognostics. Diagnostics is to investigate or analyze the cause or nature of a condition, situation, or problem, whereas prognosis is to know before, to calculate or predict the future as a result of rational study and analysis of available pertinent data (Cafeo, 2001). In terms of the relationship between prognostics and diagnostics, diagnostics is when a failure mode is detected within a system or subsystem; prognostics occurs when a small incipient fault is detected, and a relatively accurate estimation of useful life remaining and/or performance life remaining until full failure is determined. (Hess, 2002).

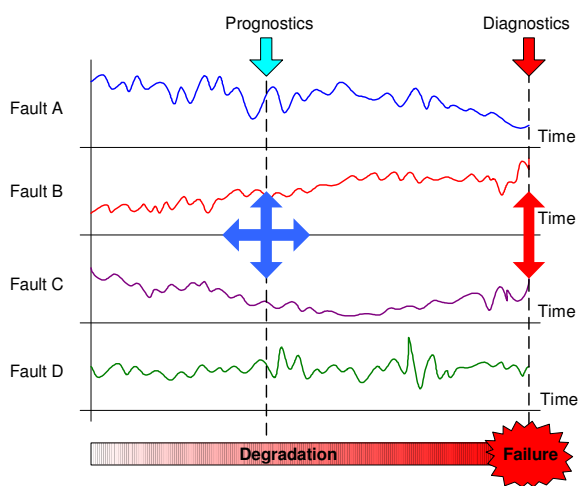

Fig. 1. Different perception of diagnostics and prognostics.

As shown in Figure 1, faults A, B C and D represent impending faults in a machine. After a long 
degradation stage where initial defects are developing, fault B finally caused the machine to break down. Diagnostics can be conceptualized as a vertical exploration of this problem to identify the leading cause after the failure has occurred, while prognostics is to investigate the problem both vertically and horizontally during the degradation period. The key concept behind prognosis is to predict an event before its possible occurrence. Time is thus inherent to the concept of prognosis and distinguishes it from that of diagnosis, where the future plays a less important role. Prognostics not only intends to discover the potential failure mode, but also to forecast the development pattern and tendency of that fault.

The architecture of diagnostic and prognostic system frameworks should be different because of their different objectives. A typical diagnostic system might consist of a data collection system, signal processing, feature extraction module, and knowledge base of faults, which may be derived from expert knowledge, physical models, and historical data. The final reasoning decision is made by comparing the feature extraction result with the knowledge base. The diagnosis is essentially a pattern recognition method that answers the fundamental question, "what is the problem?"

On the other hand, in order to conduct the prognosis, several other steps are required. In addition to the feature extraction and knowledge base of faults, which can be inherited from diagnostics, other modules are required, including performance assessment, degradation models, failure analysis and health management, and prediction. Integrating performance assessment and degradation models describes a machine's relative health status and indicates what kind of degradation patterns may exist. Prediction algorithms, which could be derived from classical time series analysis theories, statistics, and artificial intelligent technology, forecast when the machine performance will decrease to an unacceptable level as defined by the failure analysis and health management modules.

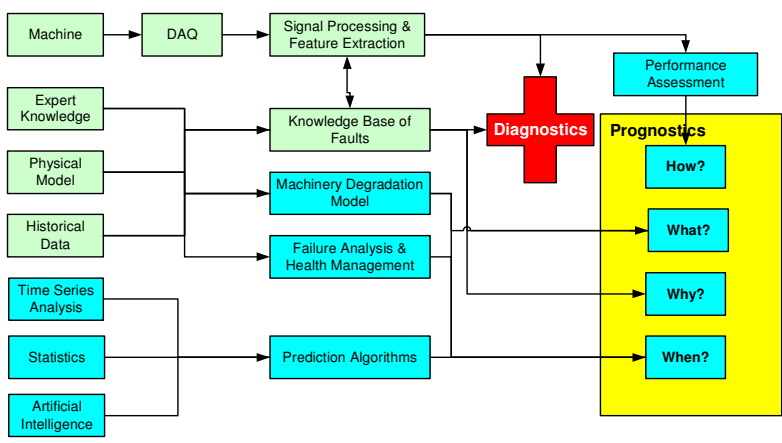

Fig. 2. System framework of a diagnostic and prognostic system.

\section{THE STATE OF THE ART OF PROGNOSTICS}

For many years, prognostic methods have been successfully developed and utilized in medical applications. Medical prognosis is defined as the prediction of the future course and outcome of disease processes, which may either concern their natural course or their outcome after treatment (Abu-Hanna, 2001). Derived from the same concept as medical prognostics, a lot of prognostic methods and systems have been developed for machinery maintenance in the past twenty years. Vibration signature analysis and oil analysis, because of their excellent capability of describing machine performance, have been employed for prognostics for a long time. Today, many prognostic applications are still based on those classical methods. (Liang, 1988, Hardman, 2000) Other measurement approaches, such as time/stress, temperature, acoustic emissions, and ultrasonics are widely employed as well. Sensor fusion techniques are also commonly engaged due to the inherent superiority in taking advantage of multiple sensor information.(Roemer,2001, Hansen, 1994, Reichard, 2000)

A large variety of prognostic applications have been reported in the technical literature as well. Prognostic approaches, mainly for gas turbine applications, are reviewed and classified as experienced-based, evolutionary, feature progression and AI-based, state estimator, and physics-based prognostics. (Byington, 2003). Generally speaking, current prognostic approaches can be categorized into three basic clusters, including model-based prognostics, data driven prognostics, and hybrid prognostics systems. An example of a characteristic model-based prognostics application includes data collected from model-based simulations under nominal and degraded conditions (Luo, 2003). In the absence of a reliable or accurate system model, another approach to determine the remaining useful life is to monitor the trajectory of a developing fault and predict the amount of time until the developing fault reaches a predetermined level requiring action, which is the so called data driven prognostic method. The Alpha-Beta-Gamma tracking filter and the Kalman filter, two well-known tracking and prediction tools, have been applied to gearbox prognostics (McClintic, 1998, Ferlez, 1998). Both filters have been investigated for their ability to track and smooth features from gearbox vibration data. An example of a hybrid method, which fuses the modelbased information and sensor-based information and takes advantage of both model-driven and data-driven methods, was proposed by Hansen (1994), by which a more reliable and accurate prognostic result can be generated.

Although the development of technology has lead to the recognition that prognostics is both desirable and technically feasible, and preliminary successful stories give us more confidence and anticipation about this emerging technique, there are still several key issues that are not entirely solved:

- Most of the developed prognostics approaches are for specific applications or equipments. A clear 
prognostic methodology for a generic machinery does not exist.

- Current methods are normally focused on solving the component or part degradation problem. A system performance assessment and degradation model is desired.

- Features used for prognostics may be different from those used for diagnostics. Enhanced feature extraction methods for the purpose of prognostic need to be further investigated.

- A variety of prediction algorithms exist in academia and perform very well for simulation signals, but their performance is not satisfactory for industrial applications due to the inherent signal complexity and low signal to noise ratio condition in real industrial environments.

Aiming to address these issues, a prognostic "toolbox" approach, called Watchdog Agent ${ }^{\mathrm{TM}}$, is being developed by the Center for Intelligent Maintenance Systems (IMS). (Djurdjanovic, 2003) In the following sections, an overall prognostic methodology will be presented. Efforts and advances of how the key issues are addressed respectively will be introduced accompanied with case studies.

\section{IMS PROGNOSTIC APPROACHES}

\subsection{Prognostic System Architecture}

As described in the pervious section, the fundamental objectives of prognostics are (a) to predict the remaining useful life and (b) to predict the machine failure modes. Based on this scheme, a prognostic system architecture can be constructed as depicted in Figure 3.

The simplified system consists of two processes. One process is the system confidence value calculation, which yields a overall system performance assessment. The other process is the prognostics in the feature domain (Engel, 2000, Djurdjanovic, 2003), which is designed to reveal the impending failure modes and the remaining useful life. Two processes could be either parallel or sequential, depending on system requirements. In the confidence value domain, firstly, a signal relevance study will be conducted for the multiple sensor inputs. Signals will be clustered and weighed according to its relationship. Secondly, a performance assessment algorithm, which embeds the sensor fusion mechanism, will transform the signals into a system level confidence value that depicts the machine's overall health status. The calculation of confidence value should be calibrated by the historical failure analysis or physical life cycle model, which ensures that a reasonable threshold can be defined. Finally, confidence value can be tracked and predicted using time series analysis methods. In the feature domain, with the exception of abrupt catastrophic failures, most of the faults have some kind of progression process to failure. Consequently their coordinates in feature space should have traceable trajectories drifting from the normal operation region to various fault regions. By continuously tracking the trajectories, prognostics in feature space can be conducted. The challenge to prognostics is how to extract effective features that can construct a reasonable feature space. This question is further explored in the next section.

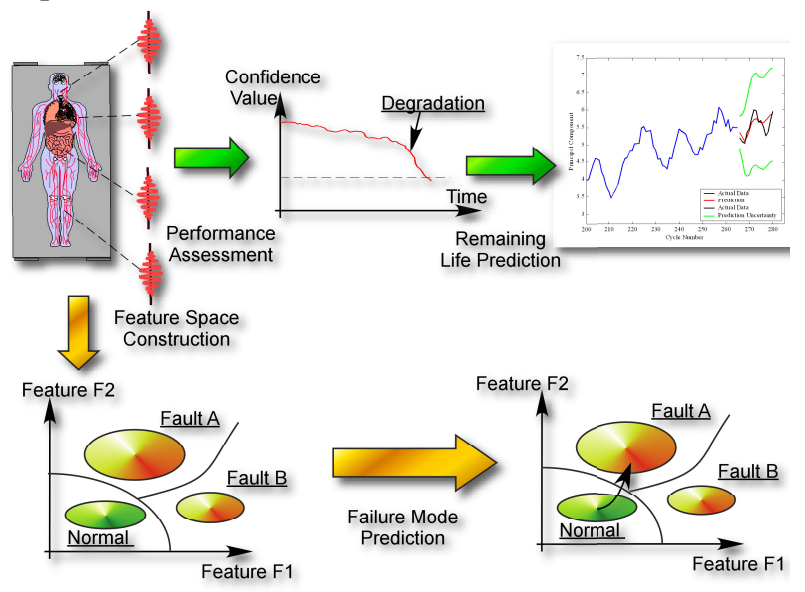

Fig. 3. IMS prognostic system architecture.

\subsection{Feature Extraction}

\section{Weak Signature Detection}

Early stage fault detection is one of the most difficult tasks prognostics has to confront. At the initial stage of an impending fault, the defect symptoms are difficult to detect because of its weak predominance compared with the background noise and other interferences. Methods that can extract the weak signature of incipient defects are essential to early defect detection. A wavelet filter based weak signature detection method is developed by Center for IMS developed. Its successful applications in gearbox and rolling element bearing early defect detection are reported by Lin (2003) and Qiu (2004).

\section{Construction of Feature Space}

The ideal situation for prognostics is that with the assistance of historical data and expert knowledge, all the possible failure modes can be located and labeled in a feature space so that a clear trajectory of fault progression is achievable. Several powerful methods can be used to construct the feature space, such as Principal Component Analysis (PCA), Self Organized Map (SOM), and Support Vector Machine (SVM)., etc. However, quite often the prior knowledge is very limited or not applicable due to data constraints and variation of operational conditions. Therefore, an overall feature space representation is not always available. One of the approaches to address this issue is to develop autonomous learning mechanism, such as the Advanced Resonance Theory (ART2)-based learning tool (Carpenter, 1987), to learn and identify unknown feature incrementally.

\subsection{Performance Assessment Methods}

One of the key issues for prognostic problems is how to assess the system performance and establish a 
generic degradation model. Watchdog Agent ${ }^{\mathrm{TM}}$ is based on utilizing the performance-related information obtained from the signatures extracted from multiple sensor inputs. The performance assessment is achieved based on matching of the signatures representing the most recent performance with those observed during the normal system behavior. High matching among these signatures would indicate good performance, while a decreasing trend of matching would indicate performance degradation and the need for maintenance. A performance assessment module evaluates the overlap between the most recently observed signatures and those observed during the normal state of operation. This overlap is expressed through the so-called Confidence Value (CV), with higher CVs signifying a high overlap, and hence performance closer to normal. In case signals, or data associated with some failure mode exists, most recent performance signatures obtained through the signal processing, feature extraction, and sensor fusion modules can be matched to signatures extracted from faulty behavior data sets. This matching enables recognition and forecasting of a specific faulty behavior.

Realization of the performance evaluation module depends on the character of the application and extracted performance signatures. If significant application expert knowledge exists, simple but rapid performance assessment based on the feature-level fused multi-sensor information can be made using the relative number of activated cells in the CMAC neural network (Albus, 1975), or using the logistic regression approach (Yan, 2002). For open-control architecture products, the match between the current and nominal control inputs and performance criteria can also be utilized to assess the product's performance. (Tong, 2002) For more sophisticated applications with complicated signals and performance signatures, statistical pattern recognition methods (Djurdjanovic, 2002), or feature map-based approach (Casoetto, 2003) can be adopted. Two selected performance assessment methods of the Watchdog Agent ${ }^{\mathrm{TM}}$ toolbox are presented in the following section.

\section{Model Based System Performance Assessment}

For a linear time-invariant (LTI) system, the dynamic parameters do not change regardless of the input signal applied to the system or the operating point of the system. (Dailly , 1990) However, the dynamic system parameters will almost certainly change in some way in response to the development of a fault. If a system model can be establish by parameter estimation techniques, the residual of the system output and model output can be calculated thereafter. Accordingly, the residual presents a way for assessing the system model.

As shown in Figure 4, $u(t)$ is the input to the system; $y(t)$ is the output from the system before the addition of measurement noise $\mathrm{n}(\mathrm{t}) ; y_{m}(t)$ is the measurement output of the system; $\hat{y}(t)$ is the system output estimated by the system model; $r(t)$ is the residual that shows the system drift. If the system model accurately represents the dynamic system, the residual $r(t)$ will reflect the measurement noise, which is assumed to be white Gaussian noise. However, if the system is changing because of the development of impending faults, the residual $r(t)$ will reflect how much the system has changed with respects to its original condition as represented by the estimated (healthy) system model. This method has been successfully demonstrated on an Anti-Lock Braking System (ABS) by Tong (2002). The challenge of this approach is how to construct a robust system model that can accommodate noise and multiple operation points. The system model must be able to distinguish the system degradation and process changes. For a nonlinear or time-variant system, parameter space bounds are very complex and would not lend themselves to simple computation, and therefore require more sophisticated system identification and modeling methods.

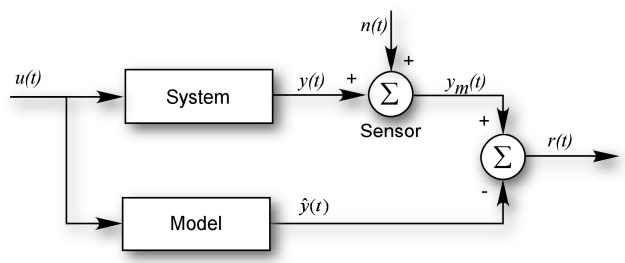

Fig. 4. Residual calculation based on a system model.

\section{Logistic Regression Method}

The machine condition description from daily maintenance records and $\operatorname{logs}$ is a dichotomous problem (either normal or failure) which can be represented using a logistic regression function. The goal of logistic regression is to find the best fitting model to describe the relationship between the categorical characteristics of dependent variables (the probability of an event occurring, constrained between 0 and 1) and a set of independent variables. The logistic regression method was used to set up the relationships between relative feature inputs and probability of failure. By using this approach, the performance of machines can be evaluated at each operational point. In addition, logistic regression models can be trained for further root cause classification based on the probability of each fault condition (Yan, 2002).

\subsection{Prediction Algorithm Development}

Even though the performance $\mathrm{CV}$ already bares significant information about the machine healthy status, additional prognostic information such as the remaining useful life and possible failure modes, can only be revealed by capturing the dynamics of the degradation and predict it over time. Currently, Autoregressive Moving Average (ARMA) modeling (Pandit, 1993), Match Matrix, Neural Network, and Support Vector Machine are methods employed to capture the dynamic behavior of performance $\mathrm{CVs}$, or extracted performance signatures.

\section{Match Matrix}


Match Matrix is new prediction tool successfully demonstrated in an IMS testbed (Liu, 2004). Match Matrix learns the system degradation pattern from previous operational runs and then predicts the current degradation tendency by measuring the similarity of features. Match Matrix method takes the most advantage of historical data and establishes the degradation model by a purely data-driven mechanism. This method exhibits its merits for some data intensive applications where large amounts of historical data are available but an explicit physical degradation model does not exist.

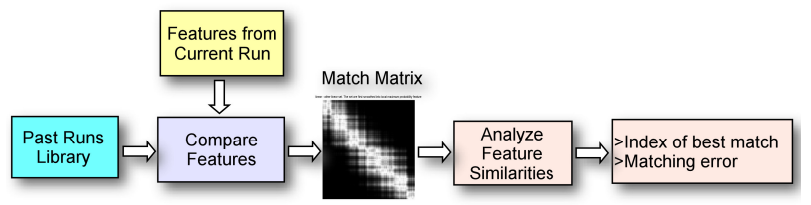

Fig. 5. Match matrix-based prediction.

\section{Neural Network}

Among various neural network structures, recurrent neural networks have shown superiority for prediction because of the capability of storing the previous states of the system through the recurrent connections. Elman Recurrent Neural Network (ERNN) is one kind of globally feed-forward, locally recurrent neural network model that is employed by the IMS Center.
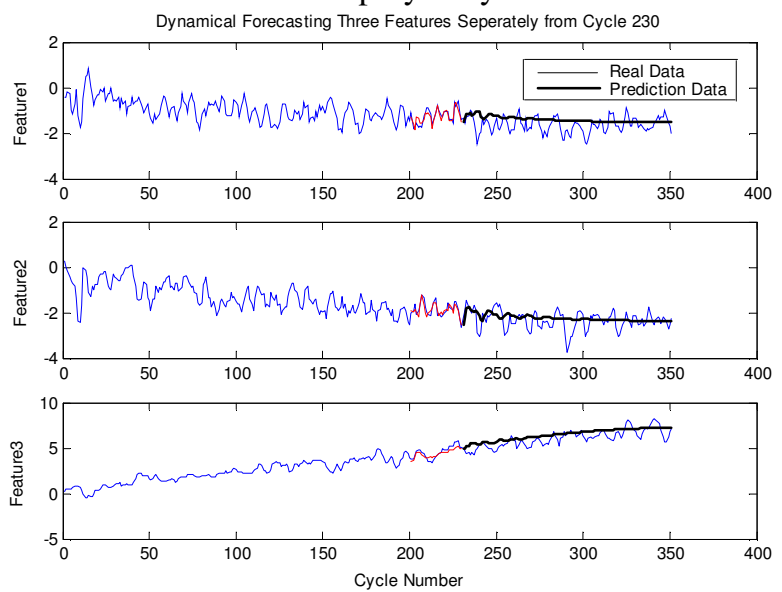

Fig. 6. Dynamic prediction using trained neural network models.

Figure 6 shows a prediction result from three features of a boring process. Load data was collected for 350 boring cycles until system degradation appeared. Joint time-frequency method and principal component analysis had been applied to generate the three features. The initial 230 cycles were used to construct the ERNN model and a 120-steps-ahead prediction has been made as represented by the dashed line. The neural network model constructed predicts the future behavior of the feature successfully.

\section{Support Vector Machine}

The foundations of Support Vector Machine (SVM) have been developed by (Vapnik, 1995) and are gaining popularity due to many attractive features and promising empirical performance. SVM has been successfully applied in classification problems. When SVM is employed to prediction, the basic idea is to feed SVM some known data and have it find a regression function for those data. In the case that the data comes from an analytical function, even if the data is contaminated with noise, SVM can still make prediction on unseen data successfully. If the data is indeterministic in an analytical way, the training strategy still works but additional efforts are required for parameter tuning of SVM. The red line of Figure 7 shows feature data of a drilling process. The first 80 data points are used for training and $1,5,10$, and 20step-ahead predictions are conducted. The prediction results are plotted in green lines. It turns out that finetuned SVM can make a good prediction on the overall trend although prediction on local variation gets worse with the step increases. Currently, more research efforts in SVM-based time series prediction are being conducted at the IMS Center.

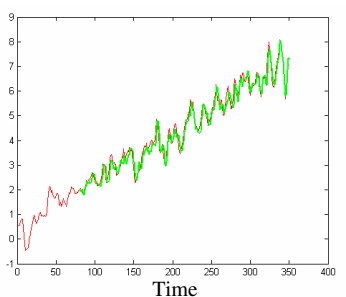

(a) one-step-ahead prediction

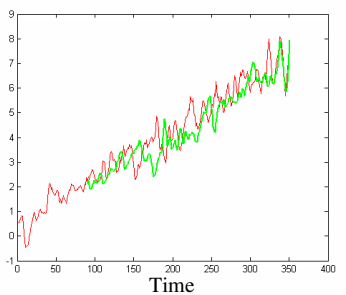

(c) ten-step-ahead prediction

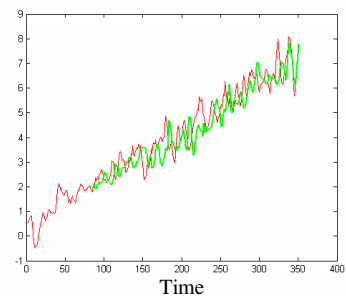

(b) five-step-ahead prediction

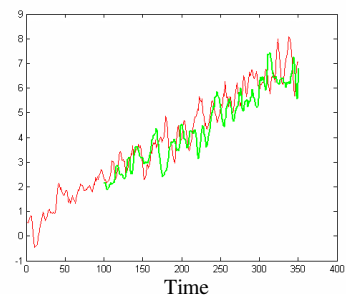

(d) twenty-step-ahead prediction
Fig. 7. The prediction result of SVM.

Due to the variation of applicability, it is quite possible that certain prediction algorithms might outperform others for some application. For example, neural network performs better to nonstationary signal than ARMA method. But its advantage is not so obvious when deals with stationary signals considering the complexity of parameter tuning and training. Match matrix method can predict the future by compare the similarity of current operation pattern with historical ones, which presumes a relative strict condition that all the system operation points are already included in the historical data. SVM method is based on the regression theory. It has unique advantage of controlling the overfitting and dealing with high noise data. But it also needs to find a solution to guide the parameter selection and tuning. So, there is no uniform method that can be directly applied to certain cases. Data interpretation and insightful understanding of methods are always very important before a method is arbitrarily selected. It is very dangerous to treat a application as a black-box problem and never attempt to understand its characteristics.

\section{CONCLUSION AND DISCUSSION}

Prognostics has higher requirements than diagnostics in feature extraction and selection methods, 
necessitating more knowledge about system degradation. Effective and robust performance assessment methods and accurate prediction algorithms are the fundamental elements of prognostics.

Performance assessment involves matching of the signatures representing the most recent performance with those observed during the normal system behavior. In real implementations, depending on the application-specific characteristics and availability of prior knowledge, a variety of performance assessment methods may need to be developed. Prediction is the final step in prognostics, and three different prediction algorithms and examples were presented in this paper. Insightful understanding of the methods and correct interpretation of the data are essential to the success of prediction. There are still a lot of unclear issues in this research field which is one of the core research directions at Center for IMS currently.

\section{ACKNOWLEDGMENT}

This research was supported by the National Science Foundation Industry/University Cooperative Research Center (NSF I/UCRC) on Intelligent Maintenance Systems (IMS) under Grant No. 0117518.

\section{REFERENCES}

Abu-Hanna, P. J. F. Lucas (2001). Prognostic Models in Medicine: AI and Statistical Approaches, Methods of Information in Medicine, Vol. 40: 1-5

Albus, J. S.(1975). A New Approach to Manipulator Control: The Cerebellar Model Articulation Controller (CMAC). Trans. of ASME, Journal of Dynamic Systems, Measurement and Control, pp. 220-227.

Byington C. S., Roemer M. J. and Galie T (2003). Prognostic Enhancements to Gas Turbine Diagnostic Systems, IEEE Aerospace Conference, Big Sky, Montana, March 2003.

Carpenter, G.A. and Grossberg, S. (1987), ART2 : Selforganization of stable category recognition codes for analog input patterns, Applied Optics, Vol.26, No.23, pp.4919-4930

Casoetto, N., Djurdjanovic, D., Mayor, R., Lee, J. and Ni, J. (2003). Multisensor Process Performance Assessment Through the Use of Autoregressive Modeling and Feature Maps. Transaction of SME, Journal of Manufacturing System, Vol. 22, pp.1-9.

Dailly,C. (1990). Fault Monitoring and Diagnosis. Computing \& Control Engineering Journal, pp.57-62

Djurdjanovic, D., Ni, J., Lee, J.(2003). Watchdog Agent-an infotronics-based prognostics approach for product performance degradation assessment and prediction, Advanced Engineering Informatics, Vol. 17, pp.109-125

Engel, S. J., Gilmartin, B. J., Bongort, K. and Hess, A., (2000), Prognostics, the real issues involved with predicting life remaining, Proc. of the IEEE Aerospace Conference Proceedings, 2000, Vol. 6, pp. 457-469

Ferlez, R.J., Lang, D.C.(1998). Gear-Tooth Fault Detection and Tracking Using the Wavelet Transform, 52nd Meeting of the MFPT

Hansen, Robert J, . Hall, David L.; Kurtz, Stewart K(1994). New approach to the challenge of machinery prognostics: Proceedings of the International Gas Turbine and Aeroengine Congress and Exposition, pp. 1-8, ASME Paper 94-GT-3
Hardman, W.; Hess, A.; Sheaffer(2000). A helicopter powertrain diagnostics and prognostics demonstration, IEEE Aerospace Conference Proceedings, v 6, pp. 355366

Hardman, W.; Hess, A.; Sheaffer, J.(1999). SH-60 helicopter integrated diagnostic system (HIDS) program-diagnostic and prognostic development experience, Proceedings of 1999 IEEE Aerospace Conference, Volume: 2 , pp. 473 491

Hess, A.(2002). Prognostics, from the need to reality-from the fleet users and PHM system designer/developers perspectives. IEEE Aerospace Conference Proceedings, Vol. 6, pp. 2791-2797

Cafeo, J. (2001). Reasoning Frameworks for Diagnosis and Prognosis, LANL Damage Prognosis Workshop

Jing Lin, M.J.Zuo (2003). Gearbox Fault Diagnosis Using Adaptive Wavelet Filter, Mechanical Systems and Signal Processing ,Vol. 17, pp. 1259-1269

Liang, E., Rodriguez, R., Husseiny, A. (1988). Prognostics/diagnostics of mechanical equipment by neural network, Neural Networks, Vol, No.1., 1988, pp.33

Liu, J., Djurdjanovic, D., Ni, J., and Lee, J. (2004), Performance Similarity Based Method for Enhanced Prediction of Manufacturing Process Performance, in Proceedings of the 2004 ASME International Mechanical Engineering Congress and Exposition (IMECE), Paper No. IMECE2004-62246, 2004

Luo, J., Namburu, M. Pattipati, K. Qiao, L. Kawamoto, M. Chigusa, S. (2003). Model-based prognostic techniques, IEEE Systems Readiness Technology Conference, 2003. pp. 330- 340

McClintic, K. (1998), Feature Prediction and Tracking for Monitoring the Condition of Complex Mechanical Systems, The Pennsylvania State University, MS Thesis, PA

Qiu, H., Lee, J., Lin, J., and Yu, G. (2003), Robust Performance Degradation Assessment Methods for Enhanced Rolling Element Bearing Prognostics, Advanced Engineering Informatics, Vol. 17, pp. 127-140

Pandit, S. M. and Wu, S-M(1993). Time Series And System Analysis With Application. Krieger Publishing Co., Malabar, FL.

Roemer, M.J. ; Kacprzynski, G.J.; Orsagh, R.F(2001). Assessment of data and knowledge fusion strategies for prognostics and health management, IEEE Aerospace Conference Proceedings, v 6, pp. 62979-62988

Reichard, Karl M.; Van Dyke, Mike; Maynard, Ken(2000), Application of sensor fusion and signal classification techniques in a distributed machinery condition monitoring system, Proceedings of SPIE - v 4051, pp. 329-336

Tong, G., Koc, M. and Lee, J.,(2002), System Performance Assessment Based on Control System Criteria Under Operating Conditions, Proc. of 5th International Conf. on Managing Innovations in Manufacturing, Milwaukee, USA.

Vapnik, V(1995). The Natural of Statistical Learning Theory. Springer-Verlag, New York, 1995

Yan, J., Koc, M., and Lee, J(2002) Predictive algorithm for machine degradation detection using logistic regression, Proc. of 5th International Conf. on Managing Innovations in Manufacturing, Milwaukee, USA. pp.171-176. 